\title{
The effect of a specific protocol to shorten the decision-to-delivery interval for emergency cesarean section
}

\author{
Dittakarn Boriboonhirunsarn $^{1}$, Prasert Sunsaneevithayakul ${ }^{1}$, and Jaruporn Teerakunwanij ${ }^{1}$ \\ ${ }^{1}$ Mahidol University Faculty of Medicine Siriraj Hospital
}

May 5, 2020

\begin{abstract}
Rationale, aims and objectives: In emergency cesarean section, time from decision to delivery should be within 30 minutes. This study aims to compare decision-to-delivery interval (DDI) in emergency cesarean section before and after the implementation of a specific care process improvement protocol ("code blue"). Methods: 300 women underwent emergency cesarean section were included. Study and comparison group were 150 women before and 150 women after "code blue" protocol implementation. Medical records were reviewed for clinical information. Timing of decision-to-delivery process was compared. Results: Maternal age, parity, and GA at delivery were comparable between the 2 groups. The most common indication was abnormal FHR in NICHD category III in both groups. Median DDI was significantly shorter in study than comparison group (22 vs. 52.5 minutes, $\mathrm{p}<0.001)$. In addition, median decision-to-room and decision-to incision intervals were also significantly shorter $(8$ vs. 25 minutes and 18 vs. 45 minutes, $\mathrm{p}<0.001$, respectively). Women in study group had significantly higher rate of DDI [?]30 minutes than in comparison group ( $80 \%$ vs. $8 \%, \mathrm{p}<0.001)$. Similar significant differences of each time interval and rate of DDI [?]30 minutes between the 2 groups were observed regardless of decision time. Only 5 (3.3\%) of women in study group had DDI $>75$ minutes compared to 13 cases $(25 \%)$ in comparison group $(\mathrm{p}<0.001)$. Pregnancy and neonatal outcomes were comparable between the 2 groups. Conclusion: The implementation of "code blue" protocol for emergency cesarean section results in significantly shorter DDI and other time intervals.
\end{abstract}

\section{Introduction}

The American College of Obstetricians and Gynecologists (ACOG) and the Royal College of Obstetrics and Gynecology (RCOG) suggest that the time from decision of emergency cesarean section to the birth of the baby (Decision-to-delivery interval, DDI) should be within 30 minutes. ${ }^{1,2}$ However, in actual clinical practice, achieving the 30-minute goal is challenging in many settings from many reasons. ${ }^{3-5}$ In addition, there is no firm evidence to confirm that DDI within 30 minutes improve maternal and neonatal outcomes. ${ }^{6-8}$ However, it is recommended that cesarean section should be carried out within 30 minutes when there is immediate threat to life of the woman or fetus, and within 75 minutes for those with maternal or fetal compromise that is not immediately life-threatening. The 30- and 75-minute goals are also recommended for measuring the performance of an obstetric unit. ${ }^{9}$

In developed countries, achievement of 30-minute goal for emergency cesarean deliveries was approximately $40 \%-65 \%{ }^{4,5,10}$ On the other hand, the achievement has been reported to be lower than $20 \%$ in developing countries. ${ }^{11-13}$ In order to achieve the 30-minute goal, there are many challenges that may be related to logistics, patients, anesthetic, obstetric condition, communication and teamwork, depending on the context of each setting. ${ }^{14,15}$ In general, care improvement process and better preparedness can possibly reduce DDI, such as re-locating operating and delivery rooms, availability of staff team, and effective communication and teamwork. ${ }^{16}$

A previous study in Siriraj Hospital showed that only 3.5\% of women underwent emergency cesarean section 
had DDI [?] 30 minutes, while $52.0 \%$ had DDI $>75$ minutes. ${ }^{17}$ Another study in women with non-reassuring FHR also showed that only $6.6 \%$ achieved the 30 -minute goal. ${ }^{18}$ Better performance was reported in women with abnormal FHR in NICHD category III and during after office hours.

Accordingly, a multidisciplinary team, including, obstetricians, anesthesiologist, pediatricians, and other related personnel, has developed a care process improvement protocol called "code blue" in order to shorten the DDI for emergency cesarean section. The protocol includes improvement in various process including decision for cesarean section, team communication, patient preparation and transfer, and team preparedness and readiness. Conditions for emergency cesarean section have been clearly defined, which are abnormal FHR in NICHD Category III, hypovolemic shock, amniotic fluid embolism, cord prolapsed, and uterine rupture. Awareness has been raised among all staff members regarding the importance of the 30-minute goal. Communication with the staff and operating room team is initiated immediately at the time of decision together with patient preparation. Transfer process is also facilitated. During after office hours, when necessary, an extra operating room and nurse team will be made available. The "code blue" protocol has been approved and implemented in 2017 and the new workflow and instructions were explained and distributed to every staff and related personnel in the Department.

However, there has been no concrete evaluation of improvement in DDI after the implementation of "code blue" protocol. Therefore, this study was conducted to compare the DDI and other time intervals for emergency cesarean section before and after the "code blue" protocol implementation. Factors associated with delayed time intervals and pregnancy outcomes were also evaluated.

\section{Methods}

With the approval of Siriraj Institutional Review Board, a retrospective cohort study was conducted at the Department of Obstetrics and Gynaecology, Faculty of Medicine Siriraj Hospital, which is the largest university-based tertiary hospital in Thailand. A total of 300 pregnant women underwent emergency cesarean section were included. Study group consisted of 150 pregnant women underwent emergency cesarean section after implementation of the "code blue" protocol (during 2017-2018) and comparison group consisted of another 150 women before the implementation (during 2015-2016). Indications for emergency cesarean section included in this study were according to current institutional guideline which are abnormal fetal heart rate in NICHD Category III, hypovolemic shock, amniotic fluid embolism, umbilical cord prolapsed and uterine rupture.

Medical records were reviewed and related clinical information were extracted, including maternal characteristics, antenatal and intrapartum characteristics, indications for cesarean section, and neonatal outcomes. Time from decision until delivery in each woman was evaluated. Time intervals were determined, including decision-to-room interval (DRI), decision-to-incision interval (DII), and decision-to-delivery interval (DDI). The DDIs were classified into 3 groups, which are [?] $30,31-75$, and $>75$ minutes. ${ }^{1,2,9}$

Descriptive statistics were used to describe various characteristics. Comparisons between groups were performed using Student's t-test, Mann-Whitney U test, and chi square test, as appropriate. Subgroup analysis was also performed according to time of decision, either during or after office hours. P-values less than

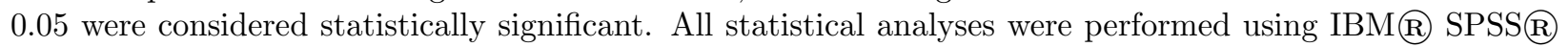
Statistics version 24 (IBM Corp., Armonk, NY, USA).

\section{Results}

Of 300 women underwent emergency cesarean section; 150 had their cesarean section before (during 20152016) and another 150 after (during 2017-2018) implementation of the "code blue" protocol.

Table 1 shows comparison of baseline characteristics between the 2 groups. Both groups were comparable with regard to maternal age, GA at delivery, and parity. The most common indication for emergency cesarean section in both groups were abnormal FHR in NICHD category III (95.3\% vs. $82 \%$ in before and after "code blue" group, respectively) and majority of decisions were made during office hours ( $65.3 \%$ vs. $64.7 \%$ in before 
and after "code blue" group, respectively). However, general anesthesia was significantly more common in women after than before "code blue" group ( $94 \%$ vs. $28 \%$, respectively, p<0.001).

Table 2 shows the comparison of various time intervals after decision of cesarean section between the 2 groups. The median time interval for every process was significantly lower in the group after "code blue" compared to those before "code blue" (DRI: 8 vs. 25 minutes, $\mathrm{p}<0.001$; DII: 18 vs. 45 minutes, $\mathrm{p}<0.001$ ), DDI: 22 vs. 52 minutes, $\mathrm{p}<0.001)$. In addition, the group after "code blue" was significantly more likely to have DDI of $<30$ minutes than those before "code blue" group ( $80 \%$ vs. $8 \%$, respectively, $\mathrm{p}<0.001)$. Only $3.3 \%$ of after "code blue" group had DDI of $>75$ minutes compared to $28.7 \%$ in before "code blue" group.

Table 3 shows the comparison of various time intervals after decision of cesarean section between the 2 groups according to time of decision. Every time interval was significantly shorter among those in after than before "code blue" group $(\mathrm{p}<0.001)$ both when decision was made during office and after office hours. Within both before and after "code blue" groups, each time interval, as well as proportion of women with DDI of [?]30 minutes were not significantly different between decision made during office and after office hours.

Table 4 shows comparison of pregnancy outcomes between the 2 groups. Both groups had comparable neonatal outcomes in terms of birth weight, Apgar scores, and NICU admission.

\section{Discussion}

Significantly shorter time intervals from decision to delivery was observed after implementation of "code blue" protocol. As many as $80 \%$ of cases had DDI [?]30 minutes, and only $3.3 \%$ had DDI $>75$ minutes after implementation of "code blue" protocol, compared to $8 \%$ and $28.7 \%$ before "code blue", respectively $(\mathrm{p}<0.001)$. The significant improvement was observed in every process after decision of cesarean section. Median DRI decreased from 25 to 8 minutes, DII decreased from 45 to 18 minutes and DDI decreased from 52 to 22 minutes (all p values <0.001). The improvements were observed in both during office and after office hours without significant differences between the different time of decision. This was different from previous reports from the same hospital that DDI was significantly shorter during after office hours ${ }^{17,18}$ and confirms the success.

The results reflect the success of "code blue" protocol implementation as DDI was significantly shortened. This was similar to other previous studies which demonstrated that various quality improvement programs, including continuous education and team training course for obstetric and related staff with emphasis on the importance of achieving the standard of 30-minutes goal, can significantly shortened DDI and other processes. ${ }^{16,19-21}$ Among many reasons for the success of "code blue" protocol was that the protocol was developed based on the evidence from previous studies in the same hospital and adjusted to the context of the hospital's workflow. Involvement of multidisciplinary team members also helps making the protocol possible and achievable after implementation in the real situation. In addition, collaboration of all related staff results in protocol compliance after implementation.

Although majority of the women achieved the 30 minutes goal, it should be noted that the were still 5 cases with DDI of $>75$ minutes and all occurred during after office hours. The delay in these cases were due to limited resources during after office hours. Although with one extra operating room and extra staff team for "code blue" situation, all were occupied by other cases of similar but more serious conditions at the same time with these 5 cases. However, these cases were initially resuscitated and provided with close monitoring and ended up with favorable outcomes.

It can be observed that there was a shift in anesthetic methods from $28 \%$ general anesthesia to $94 \%$ after "code blue" which could partly help in reducing DDI, which was similar to a previous report that general anesthesia significantly shorten DDI compared to spinal anesthesia. ${ }^{22}$ Nonetheless, a recent study reported that in optimized organization short DDI of [?]15 minutes was independent of the anesthetic technique performed. ${ }^{23}$ However, adverse neonatal outcomes associated with general anesthesia should be aware of and early insertion of an epidural catheter should be considered whenever there is a potential concern of emergency cesarean section. 
Whether short DDI improves neonatal outcomes is still controversial. A previous systematic review reported that among cesarean deliveries with immediate threat to life of the woman or fetus, no association was observed between 5 -minute Apgar score $<7$, umbilical artery $\mathrm{pH}<7.1$, and NICU admission and shorter delivery intervals. ${ }^{6}$ A more recent study also reported that decision-to-incision time of $>30$ minutes were not associated with worse maternal or neonatal outcomes. ${ }^{8}$ Previous studies from the same hospital also did not find such association between DDI and adverse neonatal outcomes. ${ }^{17,18}$ Similar results were observed in this study as well that no significant differences in adverse neonatal outcomes were observed even when DDI was shortened. However, there are many other factors that might not be measurable that are possibly related to adverse neonatal outcomes. Further studies are needed to evaluate such association.

The strengths of this study include that the intervention protocol was applied in the same setting and comparison group was selected from immediate years before the intervention that differences in other related care process should not vary significantly. Indications for cesarean section in comparison group were reviewed and only those with similar indications and diagnoses to the "code blue" protocol were included. Some limitations may include that contribution of actual component to the significant improvement as well as some other details related to the delay were not measured, e.g., transfer process details during different time of decision, anesthetic difficulties, differences between cesarean section indications, etc. Generalization of the results to other different settings might also be limited but similar quality improvement process is encouraged.

The results of this study showed that the implementation of "code blue" protocol can significantly shorten time to delivery in women requiring emergency cesarean section. However, to maintain and improve the performance of obstetric unit, the protocol needs to be audited and evaluated regularly to evaluate its performance and compliance as well as determine potential barriers that needs timely responses. Further studies are still needed to determine the rooms for improvement regarding cesarean section in cases with immediate threat to life of the woman or fetus, not only to shorten delivery time but also to improve maternal and neonatal outcomes.

\section{Conflict of interest}

None.

\section{References}

1. American Academy of Pediatrics, Americal College of Obstetricians and Gynecologists. Guidelines for Perinatal Care. 5 ed. Elk Grove Village: AAP; Washington, DC: ACOG; 2002.

2. Royal College of Obstetrics and Gynaecology. Report of a joint working group: organization standards for maternity services. London: RCOG Press; 1995.

3. Bruce D, Stone S, Harding K. Evaluation of emergency caesarean sections-completion of the audit cycle in a Central London teaching hospital. J Obstet Gynaecol. 2002;22(3):273-278.

4. Helmy WH, Jolaoso AS, Ifaturoti OO, Afify SA, Jones MH. The decision-to-delivery interval for emergency caesarean section: is 30 minutes a realistic target? BJOG. 2002;109(5):505-508.

5. Livermore LJ, Cochrane RM. Decision to delivery interval: a retrospective study of 1,000 emergency caesarean sections. J Obstet Gynaecol. 2006;26(4):307-310.

6. Tolcher MC, Johnson RL, El-Nashar SA, West CP. Decision-to-incision time and neonatal outcomes: a systematic review and meta-analysis. Obstet Gynecol. 2014;123(3):536-548.

7. Tomlinson JH, Lucas DN. Decision-to-delivery interval: Is 30 min the magic time? What is the evidence? Does it work? Best Pract Res Clin Anaesthesiol. 2017;31(1):49-56.

8. Grobman WA, Bailit J, Sandoval G, et al. The association of decision-to-incision time for cesarean delivery with maternal and neonatal outcomes. Am J Perinatol. 2018;35(3):247-253.

9. Royal College of Obstetrics and Gynaecology. NICE: Clinical Guideline on Caesarean Section. London: RCOG Press.; 2011.

10. Bloom SL, Leveno KJ, Spong CY, et al. Decision-to-incision times and maternal and infant outcomes. Obstet Gynecol. 2006;108(1):6-11. 
11. Bello FA, Tsele TA, Oluwasola TO. Decision-to-delivery intervals and perinatal outcomes following emergency cesarean delivery in a Nigerian tertiary hospital. Int J Gynaecol Obstet. 2015;130(3):279283.

12. Chukwudi OE, Okonkwo CA. Decision-delivery interval and perinatal outcome of emergency caesarean sections at a tertiary institution.Pak J Med Sci. 2014;30(5):946-950.

13. Onah HE, Ibeziako N, Umezulike AC, Effetie ER, Ogbuokiri CM. Decision - delivery interval and perinatal outcome in emergency caesarean sections. J Obstet Gynaecol. 2005;25(4):342-346.

14. Chauleur C, Collet F, Furtos C, Nourrissat A, Seffert P, Chauvin F. Identification of factors influencing the decision-to-delivery interval in emergency caesarean sections. Gynecol Obstet Invest. 2009;68(4):248-254.

15. Leung TY, Lao TT. Timing of caesarean section according to urgency.Best Pract Res Clin Obstet Gynaecol. 2013;27(2):251-267.

16. Weiner E, Bar J, Fainstein N, et al. The effect of a program to shorten the decision-to-delivery interval for emergent cesarean section on maternal and neonatal outcome. Am J Obstet Gynecol.2014;210(3):224 e221-226.

17. Khemworapong K, Sompagdee N, Boriboonhirunsarn D. Decision-to-delivery interval in emergency cesarean delivery in tertiary care hospital in Thailand. Obstet Gynecol Sci. 2018;61(1):48-55.

18. Boriboonhirunsarn D, Watananirun K, Sompagdee N. Decision-to-delivery interval in pregnant women with intrapartum non-reassuring fetal heart rate patterns. J Eval Clin Pract. 2016;22(6):998-1002.

19. Iitani Y, Tsuda H, Ito Y, et al. Simulation training is useful for shortening the decision-to-delivery interval in cases of emergent cesarean section. J Matern Fetal Neonatal Med.2018;31(23):3128-3132.

20. Fuhrmann L, Pedersen TH, Atke A, Moller AM, Ostergaard D. Multidisciplinary team training reduces the decision-to-delivery interval for emergency Caesarean section. Acta anaesthesiol Scand. 2015;59(10):1287-1295.

21. Nageotte MP, Vander Wal B. Achievement of the 30-minute standard in obstetrics-can it be done? Am J Obstet Gynecol.2012;206(2):104-107.

22. Hein A, Thalen D, Eriksson Y, Jakobsson JG. The decision to delivery interval in emergency caesarean sections: Impact of anaesthetic technique and work shift. F1000Res. 2017;6:1977.

23. Bidon C, Desgranges FP, Riegel AC, Allaouchiche B, Chassard D, Bouvet L. Retrospective cohort study of decision-to-delivery interval and neonatal outcomes according to the type of anaesthesia for code-red emergency caesarean sections in a tertiary care obstetric unit in France. Anaesth Crit Care Pain Med. 2019;38(6):623-630.

Table 1 Comparison of baseline characteristics between the 2 groups

\begin{tabular}{llll}
\hline Time intervals & $\begin{array}{l}\text { Before Code Blue } \\
\text { N=150 }\end{array}$ & $\begin{array}{l}\text { After Code Blue } \\
\mathbf{N = 1 5 0}\end{array}$ & P value \\
\hline Mean birth weight + & $\begin{array}{l}\text { Mean + SD } \\
2900.5+479.4\end{array}$ & $\begin{array}{l}\text { Mean }+ \text { SD } \\
2772.9+722.3\end{array}$ & 0.074 \\
SD (g) & $\mathbf{N}(\%)$ & $\mathbf{N}(\boldsymbol{\%})$ & \\
Apgar score $<7$ & & & \\
At 1 minute & $24(16)$ & $35(23.3)$ & 0.110 \\
At 5 minute & $10(6.7)$ & $18(12)$ & 0.112 \\
NICU admission & $9(6)$ & $15(10)$ & 0.201 \\
\hline
\end{tabular}

Table 2 Comparison of various time intervals after decision of cesarean section between the 2 groups 


\begin{tabular}{|c|c|c|c|}
\hline Time intervals & $\begin{array}{l}\text { Before Code Blue } \\
\mathrm{N}=150\end{array}$ & $\begin{array}{l}\text { After Code Blue } \\
\mathrm{N}=150\end{array}$ & $P$ value \\
\hline Time intervals & $\begin{array}{l}\text { Before Code Blue } \\
\mathrm{N}=150\end{array}$ & $\begin{array}{l}\text { After Code Blue } \\
\mathrm{N}=150\end{array}$ & $P$ value \\
\hline $\begin{array}{l}\text { Mean birth weight }+ \\
\text { SD }(g)\end{array}$ & $\begin{array}{l}\text { Mean + SD } \\
2900.5+479.4 \\
\text { N }(\%)\end{array}$ & $\begin{array}{l}\text { Mean + SD } \\
2772.9+722.3 \\
\text { N (\%) }\end{array}$ & 0.074 \\
\hline $\begin{array}{l}\text { Apgar score }<7 \\
\text { At } 1 \text { minute } \\
\text { At } 5 \text { minute } \\
\text { NICU admission }\end{array}$ & $\begin{array}{l}24(16) \\
10(6.7) \\
9(6)\end{array}$ & $\begin{array}{l}35(23.3) \\
18(12) \\
15(10)\end{array}$ & $\begin{array}{l}0.110 \\
0.112 \\
0.201\end{array}$ \\
\hline
\end{tabular}

+ Mann Whitney U test

Table 3 Comparison of various time intervals after decision of cesarean section between the 2 groups according to time of decision

\begin{tabular}{|c|c|c|c|}
\hline Time intervals & $\begin{array}{l}\text { Before Code Blue } \\
\mathrm{N}=150\end{array}$ & $\begin{array}{l}\text { After Code Blue } \\
N=150\end{array}$ & $P$ value \\
\hline & Mean + SD & Mean + SD & \\
\hline Mean birth weight + & $2900.5+479.4$ & $2772.9+722.3$ & 0.074 \\
\hline & N (\%) & N (\%) & \\
\hline Apgar score $<7$ & & & \\
\hline At 1 minute & $24(16)$ & $35(23.3)$ & 0.110 \\
\hline At 5 minute & $10(6.7)$ & $18(12)$ & 0.112 \\
\hline NICU admission & $9(6)$ & $15(10)$ & 0.201 \\
\hline
\end{tabular}

+ Mann Whitney U test

Table 4 Comparison of pregnancy outcomes between the 2 groups

\begin{tabular}{llll}
\hline Time intervals & $\begin{array}{l}\text { Before Code Blue } \\
\text { N=150 }\end{array}$ & $\begin{array}{l}\text { After Code Blue } \\
\mathbf{N}=\mathbf{1 5 0}\end{array}$ & P value \\
\hline Mean birth weight + & Mean + SD & $\begin{array}{l}\text { Mean + SD } \\
2772.9+722.3\end{array}$ & 0.074 \\
SD (g) & $2900.5+479.4$ & $\mathbf{N}(\mathbf{\%})$ & \\
Apgar score $<7$ & $\mathbf{N}(\mathbf{\%})$ & $35(23.3)$ & \\
At 1 minute & $24(16)$ & $18(12)$ & 0.110 \\
At 5 minute & $10(6.7)$ & $15(10)$ & 0.112 \\
NICU admission & $9(6)$ & & 0.201 \\
\hline
\end{tabular}

